University of Nebraska - Lincoln

DigitalCommons@University of Nebraska - Lincoln

\title{
4-1-1981
}

\section{Rare-earth-rich metallic glasses. I. Magnetic hysteresis}

George C. Hadjipanayis

University of Nebraska-Lincoln, hadji@udel.edu

David J. Sellmyer

University of Nebraska-Lincoln, dsellmyer@unl.edu

B. Brandt

Francis Bitter National Magnet Laboratory, Massachusetts Institute of Technology, Cambridge, Massachusetts

Follow this and additional works at: https://digitalcommons.unl.edu/physicssellmyer

Part of the Physics Commons

Hadjipanayis, George C.; Sellmyer, David J.; and Brandt, B., "Rare-earth-rich metallic glasses. I. Magnetic hysteresis" (1981). David Sellmyer Publications. 165.

https://digitalcommons.unl.edu/physicssellmyer/165

This Article is brought to you for free and open access by the Research Papers in Physics and Astronomy at DigitalCommons@University of Nebraska - Lincoln. It has been accepted for inclusion in David Sellmyer Publications by an authorized administrator of DigitalCommons@University of Nebraska - Lincoln. 


\title{
Rare-earth-rich metallic glasses. I. Magnetic hysteresis
}

\author{
G. Hadjipanayis and D. J. Sellmyer \\ Behlen Laboratory of Physics, University of Nebraska, Lincoln, Nebraska 68588 \\ B. Brandt \\ Francis Bitter National Magnet Laboratory, Massachusetts Institute of Technology, \\ Cambridge, Massachusetts 02139 \\ (Received 7 July 1980)
}

\begin{abstract}
An anomalous magnetic behavior has been observed in rare-earch-rich metallic glasses of the composition $\left(R_{75} \mathrm{Au}_{25}\right)_{100-x} \mathrm{~B}_{x}$ where $R$ denotes $\mathrm{Pr}, \mathrm{Gd}, \mathrm{Tb}, \mathrm{Er}$ and $x=0,10$. The observed hysteresis in all samples except $\mathrm{Gd}$ at liquid-helium temperatures is due to the large anisotropy which develops below the ordering temperature. High-field-magnetization measurements up to $220 \mathrm{kOe}$ were used to determine the spontaneous magnetization and the magnetic anisotropy constant which was found to be of order $10^{8} \mathrm{erg} / \mathrm{cm}^{3}$ for the Tb and Er glasses. These parameters were used together with the observed remanence ratio to examine the high coercive fields in terms of recently proposed models of random anisotropy.
\end{abstract}

\section{INTRODUCTION}

Recently there has been a considerable interest in the study of the magnetic properties of rare-earth amorphous materials. Most of the early studies ${ }^{1.2}$ have been directed to sputtered $R \mathrm{Fe}_{2}$ and splatcooled $^{3.4} R_{65}(\mathrm{Fe}, \mathrm{Co})_{35}$ amorphous alloys. Recent$\mathrm{yy}^{5.6}$ some results were reported on sputtered $R(\mathrm{Ag}, \mathrm{Cu})$ and splat-cooled ${ }^{7.8} R_{75} \mathrm{Au}_{25}$ alloys where a single magnetic species is present and the interpretation of the experimental results becomes easier.

The unusual magnetic properties of the previously mentioned alloys containing non- $S$-state ions can be summarized as follows: (i) Some kind of magnetic ordering âppears at low temperatures. (ii) Below the ordering temperature a large magnetic anisotropy develops causing high coercivities and lack of saturation. (iii) Around the coercive field sharp magnetization jumps are observed, and (iv) strong temperature-dependent magnetic after-effects appear at helium temperatures suggesting a thermal activation process. In an attempt to explain these properties Harris et al. ${ }^{9}$ proposed the local random anisotropy model. The model postulates that in the non- $S$ state ions local random electrostatic fields act on the localized $4 f$ shells of the rare-earth atoms and produce large anisotropy axes randomly oriented. The competing influences between the local magnetic anisotropy and exchange interaction inhibit the complete alignment of the spins and produce a canted ferromagnetic state known as "asperomagnetic" (positive $\mathcal{J}$ ) or a "speromagnetic" (negative $\mathcal{d}) .10$ Similar predictions were made by Chi and Alben ${ }^{11}$ using Monte Carlo techniques and by Patterson et al. ${ }^{12}$ using numerical studies of the mean-field approxima- tion. However, recently Chi and Egami ${ }^{13}$ and Jayaprakash and Kirkpatrick ${ }^{14}$ with more elaborate methods have shown that the ground state is spinglass-like for positive $\mathcal{O}$, thus confirming the predictions of the renormalization-group calculations by Pelcovits et al. ${ }^{15}$ Harris ${ }^{16}$ has also shown recently that the true ground state is that with about $25 \%$ of the spins reversed. It appears, therefore, that the most recent theoretical papers are concluding that the ground state is not the asperomagnetic state. In a different way Callen et al. ${ }^{17}$ tried to explain the anomalous magnetic behavior assuming the existence in amorphous materials of small regions of uniaxial magnetic anisotropy ("microdomains") with their easy axes randomly oriented.

In this paper we investigate the origin of the high coercive fields observed in $R_{75} \mathrm{Au}_{25}$ amorphous alloys. For this we used high-field magnetization measurements (up to $220 \mathrm{kOe}$ ) to determine the spontaneous magnetization and the magnetic anisotropy constant. The values of these parameters were subsequently used together with the observed remanence ratio to predict the high coercive fields at absolute zero employing models of Callen et $a l^{17}$ and Harris et al. ${ }^{9}$

\section{EXPERIMENTAL}

Alloys of nominal composition $\left(R_{75} \mathrm{Au}_{25}\right)_{100-x} \mathbf{B}_{x}$ where $R$ denotes $\mathrm{Pr}, \mathrm{Gd}, \mathrm{Tb}, \mathrm{Er}$ and $x=0,10$ were made with elemental constituents of at least $99.9 \%$ purity, using an arc furnace under argon atmosphere. The amorphous samples were prepared by the splatcooling technique. ${ }^{18} \mathrm{X}$-ray diffractometer measure- 
ments were made on thin foils with a Philips diffractometer using a Mo $K_{\alpha}$ radiation and yield typical amorphous patterns. In the $\mathrm{Pr}$ and Er alloys, 10 at. $\%$ boron was added to obtain a glassy material.

Magnetization measurements were made up to 220 $\mathrm{kOe}$ in the temperature range of 1.6 to $100 \mathrm{~K}$ using a vibrating sample magnetometer.

\section{RESULTS}

The temperature dependence of the coercive fields is shown on Fig. 1. In $\mathrm{Tb}_{75} \mathrm{Au}_{25}$ the coercivity at 1.6 $\mathrm{K}$ appears to be slightly smaller than the value at 4.2 $\mathrm{K}$. This is believed to be due to a magnetothermal effect for the following reasons. The thermal conductivity of a glass is expected to be small at helium temperatures so the magnetization processes take place adiabatically. A reversal of a group of spins alters the effective exchange local field of neighboring spins which then reverse in cascade. Such reversal releases magnetic energy which heats the surroundings. Because of the vanishingly small specific heat the surrounding regions may be heated to a temperature sufficient to activate other reversals. This process may continue until an equilibrium temperature is achieved. The magnetothermal effect is further suggested by the fact that the shape of the hysteresis loops depends on the sweeping rate of the applied field (Fig. 2). It is rather difficult to measure the coercive field because of the multiple asymmetric discontinuities on the magnetization curve. Presumably if the sweeping time is made very long the discontinuities would disappear and the loops would be symmetrical. Values of the coercive fields extrapolated to absolute zero and the remanences at $1.6 \mathrm{~K}$ are listed on Table $\mathbf{I}$.

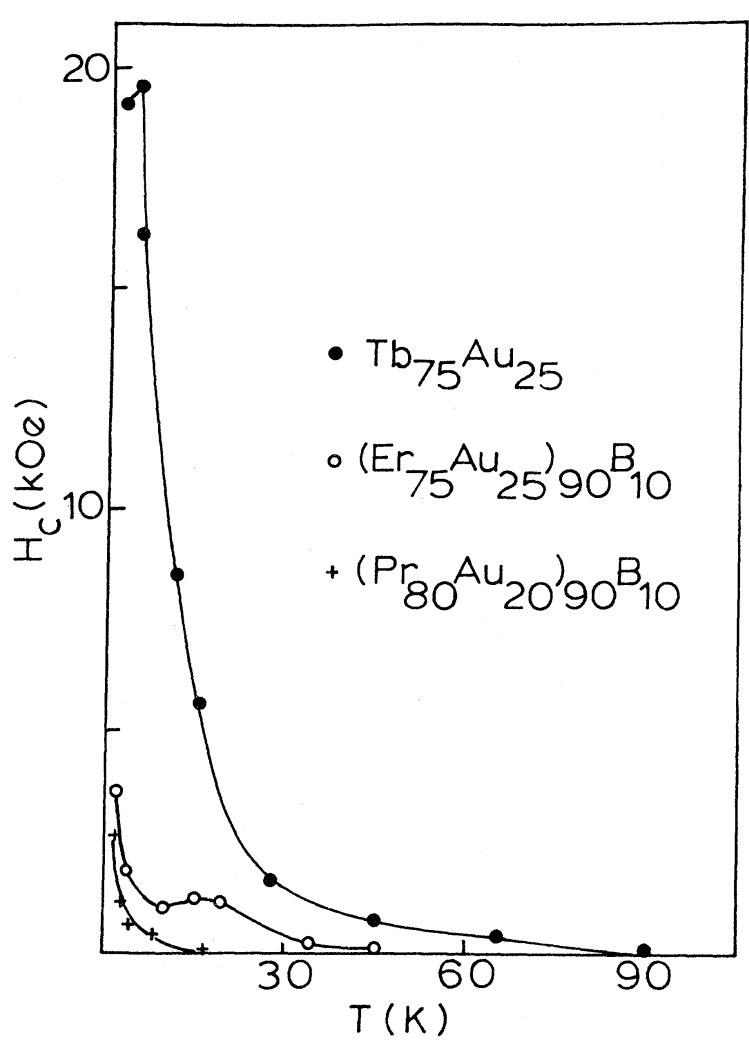

FIG. 1. Temperature dependence of coercive field in $\left(\operatorname{Pr}_{80} \mathrm{Au}_{20}\right)_{90} \mathrm{~B}{ }_{10}, \mathrm{~Tb}_{75} \mathrm{Au}_{25}$ and $\left(\mathrm{Er}_{75} \mathrm{Au}_{25}\right)_{90} \mathrm{~B} \mathrm{~B}_{10}$ glasses.

Figure 3 shows the magnetization curve in $\mathrm{Tb}_{75} \mathrm{Au}_{25}$ at $1.6 \mathrm{~K}$. It is evident that large fields are required to saturate the magnetization of the sample suggesting a large value of the magnetic anisotropy $K$.

In order to evaluate the anisotropy constant $K$ and

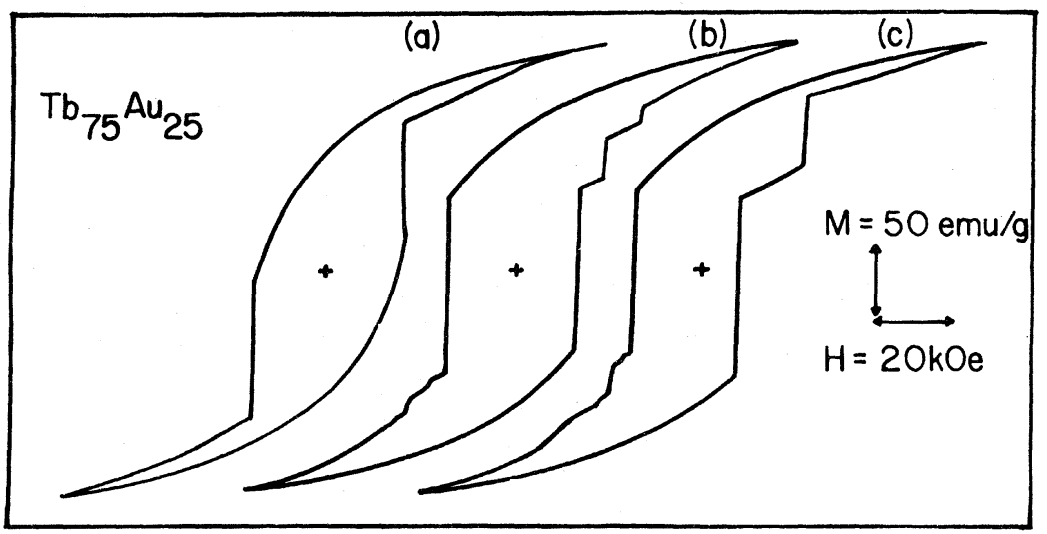

FIG. 2. Hysteresis loops in amorphous $\mathrm{Tb}_{75} \mathrm{Au}_{25}$. (a) $T=4.2 \mathrm{~K}$, sweep rate $=60 \mathrm{Oe} / \mathrm{s}$. (b) $T=1.6 \mathrm{~K}$, sweep rate $=60 \mathrm{Oe} / \mathrm{s}$. (c) $T=1.6 \mathrm{~K}$, sweep rate $=200 \mathrm{Oe} / \mathrm{s}$. 


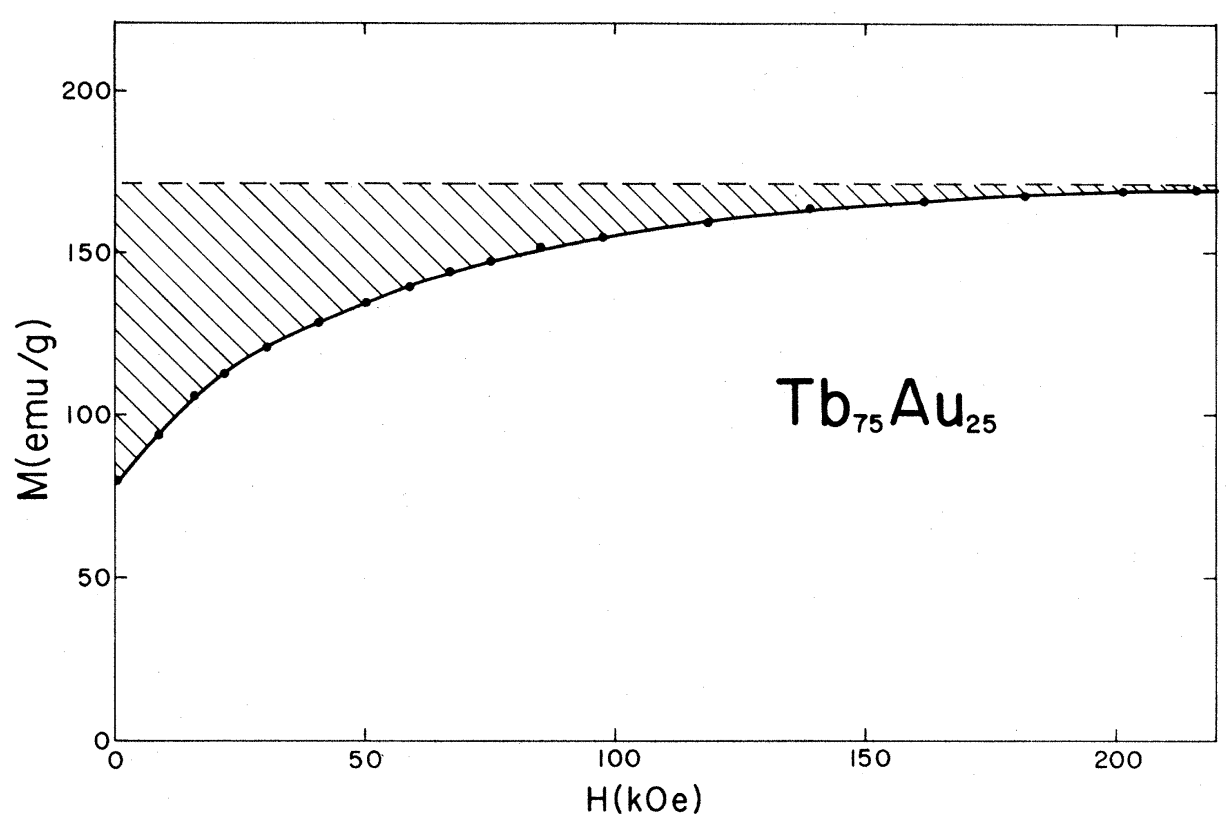

FIG. 3. Magnetization curve (field decreasing) in amorphous $\mathrm{Tb}_{75} \mathrm{Au}_{25}$ at $4.2 \mathrm{~K}$.

the spontaneous magnetization $M_{0}$, high-field magnetization measurements were made up to $220 \mathrm{kOe}$ (Fig. 4). The magnetization of $\mathrm{Gd}_{75} \mathrm{Au}_{25}$ saturates more easily than the others since $\mathrm{Gd}$ is an $S$-state ion (small anisotropy). The high-field susceptibility of the Gd glass is found to be $\chi \simeq 4 \times 10^{-5} \mathrm{emu} / \mathrm{g}$ and is very close to that of polycrystalline Gd sample. This susceptibility represents the increase of the magnetization within one domain over the spontaneous value due to redistribution in the populations of spins at high fields. Possible contributions to it come from Pauli and Van Vleck paramagnetism. ${ }^{19}$

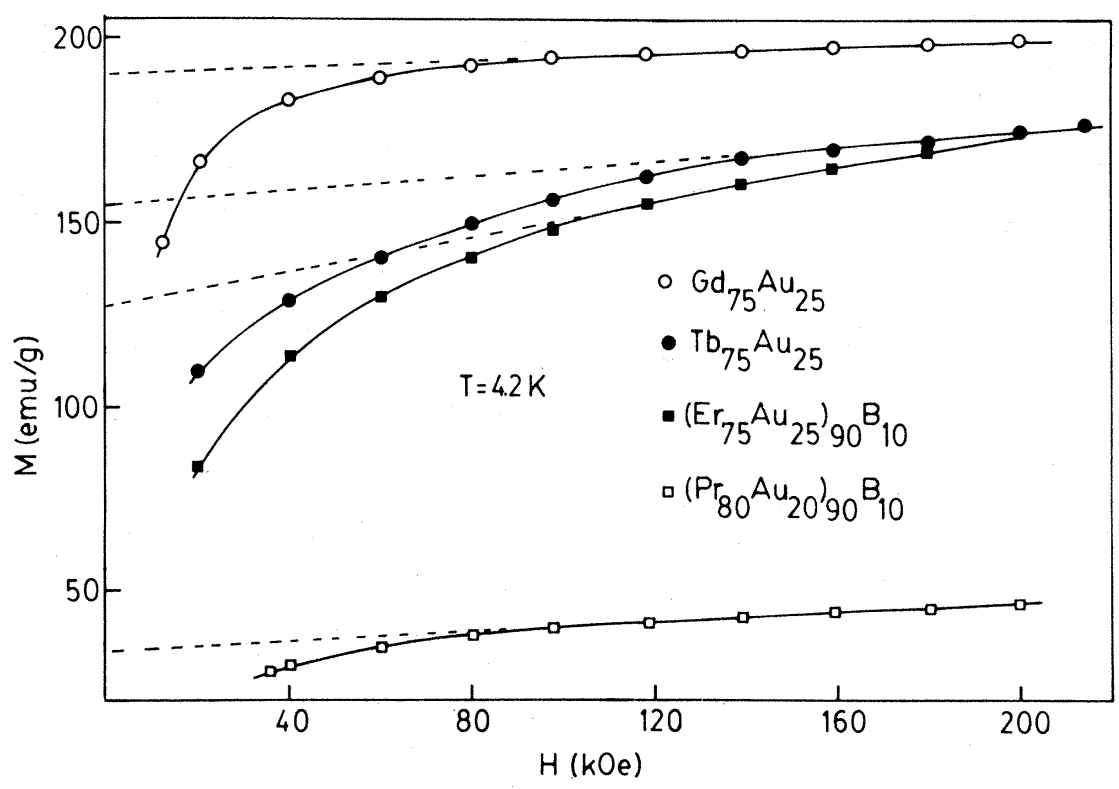

FIG. 4. High-field magnetization measurements for $R$-Au glasses. 
TABLE I. Magnetic properties of the glasses.

\begin{tabular}{lccc}
\hline \hline & $\left(\mathrm{Pr}_{80} \mathrm{Au}_{20}\right)_{90} \mathrm{~B}_{10}$ & $\mathrm{~Tb}_{75} \mathrm{Au}_{25}$ & $\left(\mathrm{Er}_{75} \mathrm{Au}_{25}\right)_{90} \mathrm{~B}_{10}$ \\
\hline$M_{S}=g J \mu_{B}(\mathrm{emu} / \mathrm{g})$ & 93.2 & 223.8 & 214.3 \\
$M_{0}(\mathrm{emu} / \mathrm{g})$ & 39 & 170 & 145 \\
$M_{R}(\mathrm{emu} / \mathrm{g})$ & 4 & 110 & 44 \\
$m_{R}$ & 0.10 & 0.65 & 0.30 \\
$\chi^{\left(10^{-5} \mathrm{emu} / \mathrm{g} \mathrm{Oe}\right)}$ & 4 & 4 & 16 \\
$K^{\mathrm{a}}\left(10^{7} \mathrm{erg} / \mathrm{cm}^{3}\right)$ & 1.7 & 8.5 & 8.9 \\
$K^{\mathrm{b}}\left(10^{7} \mathrm{erg} / \mathrm{cm}^{3}\right)$ & & 30 & 12 \\
$H_{c, 0}(\mathrm{kOe})$ & 6 & 40 & 32 \\
$H_{c, 0}^{\mathrm{c}}$ & 13 & & \\
$H_{c, 0}{ }^{\mathrm{d}}(\mathrm{kOe})$ & & &
\end{tabular}

alaw of approach to saturation.

${ }^{\mathrm{b}}$ Area between magnetization curves.

\section{DISCUSSION}

\section{A. Magnetic anisotropy}

Values of the magnetic anisotropic $K$ were found by two methods, the law of approach to saturation and magnetization-area method.

\section{Law of approach to saturation}

The high-field magnetization measurements, $M(H)$, (Fig. 4) were least-squares fitted to the usual expression $^{20}$

$$
\begin{aligned}
& M=M_{0}\left(1-\frac{A}{H^{2}}\right)+\chi H, \\
& A=\frac{4}{15} \frac{K^{2}}{M_{0}^{2}},
\end{aligned}
$$

where $M_{0}$ is the spontaneous magnetization, $\chi$ the high-field susceptibility, and $A$ a constant which is a function of $K$ and $M_{0}$.

From the best fit, values of $M_{0}, \chi$, and $A$ were obtained and subsequently were used to determine $K$ using Eq. (2). Values of the parameters obtained by this way are displayed on Table $I$. The values of the spontaneous magnetization $M_{0}$ are found to be smaller than $M_{s}=g J \mu_{B}$, the value of free ion spins corresponding to complete alignment of the moments. However in the "microdomain" model that would suggest that the spins in a domain are distributed in a cone having a net moment smaller than $M_{s}$. The values of $\chi$ obtained for $\operatorname{Pr}$ and $\mathrm{Tb}$ glasses are very similar to the observed high-field susceptibility of the soft Gd glass. However in Er glass, $\chi$ is significantly larger. This together with the very small spontaneous magnetization would suggest the existence of antiferromagnetic interactions which might
'Callen et al. model.

${ }^{\text {d}}$ Ferrer et al. model.

break up in high fields giving rise to a large $\chi$.

The values of the anisotropy constants determined are comparable with those reported by Boucher ${ }^{21}$ for equiatomic $R \mathrm{Ag}(R=\mathrm{Ho}, \mathrm{Dy}, \mathrm{Tb})$ amorphous alloys.

\section{Magnetization-area method}

This is a method ${ }^{22}$ similar to that used by Sucksmith. ${ }^{23}$ In this type of calculation one assumes that the magnetic material is composed of magnetic domains of uniaxial anisotropy $K$ randomly oriented. In an applied field $H$ the total energy of a magnetic domain of easy axis $K$ and magnetization $M_{0}$ can be written as

$$
E=K \sin ^{2}(\theta-\phi)-H M_{0} \cos \theta,
$$

where $\theta$ and $\phi$ are the angles the magnetic field makes with the magnetization and the easy axis of the domain, respectively.

At equilibrium the magnetization of the domain points in a direction which minimizes the energy $E$. This is found by differentiating Eq. (3) with respect to the angle $\theta$

$$
\frac{d E}{d \theta}=K \sin 2(\theta-\phi)-H \frac{d M}{d \theta}=0,
$$

since $M_{0} \cos \theta=M$. Equation (4) can be rearranged to

$$
\int_{0}^{M_{0}} H d M=\int_{\phi}^{0} K \sin 2(\theta-\phi) d \theta=K \sin ^{2} \phi,
$$

since at $H \rightarrow \infty, \theta=0$ and at $H \rightarrow 0, \theta=\phi$. The effect of the other domains is taken into account by a spherical average of the anisotropy energy over all possible orientations $\phi$ of the easy axes $K$,

$$
\int_{M_{R}}^{M_{0}} H d M=\left(\int_{0}^{\pi / 2} K \sin ^{2} \phi \sin \phi d \phi\right) /\left(\int_{0}^{\pi / 2} \sin \phi d \phi\right)
$$


leading to

$$
\int_{M_{R}}^{M_{0}} H d M=\frac{2}{3} K
$$

where $M_{R}$ is the average remanence of the sample.

The left-hand side (LHS) of Eq. (6) is the shaded area of the magnetization curve shown in Fig. 3. In $\mathrm{Tb}_{75} \mathrm{Au}_{25}$ the anisotropy constant found by this way $\left(K \sim 0.85 \times 10^{8} \mathrm{erg} / \mathrm{cm}^{3}\right.$ ) is surprisingly close to that found from the law of approach to saturation (Table I). It may thus be suggested that the latter method in estimating $K$ for uniaxial materials is more convenient.

\section{B. Local random anisotropy models}

The values determined for $K$ and $M_{0}$ together with the observed remanence ratio were used to examine the magnetic hysteresis properties in the light of two recently proposed models, the "microdomain" local random anisotropy model ${ }^{17}$ and the single-ion anisotropy model. ${ }^{9}$

\section{Microdomain model}

In an attempt to explain the magnetic hysteresis in amorphous $\mathrm{TbFe}_{2}$ Callen et al. ${ }^{17}$ assumed the existence, in amorphous materials, of small ordered magnetic regions (domains). of uniaxial anisotropy $K$ with the easy axes of different domains randomly oriented. Inside each domain there is a strong exchange interaction. However the interaction between different domains is assumed to be much weaker represented by a term $\lambda M$ where $\lambda$ is the interaction parameter and $M$ the average magnetization of the sample. The solution was similar to that of Stoner and Wohlfarth ${ }^{24}$ modified by an exchange-enhanced effective field, $H+\lambda M$. The model predicts reduced remanences between 1 and 0.5 (Stoner and Wohlfarth value) depending on the exchange parameter, $\lambda$. The variation of the coercive field with $\lambda^{\prime}$ is shown in Fig. $5\left(h=H M_{0} / 2 K, \lambda^{\prime}=\lambda M_{0}^{2} / 2 K\right)$.

A value of $\lambda^{\prime}$ was estimated from the experimentally measured true remanence, $m_{R}=M_{R} / M_{0}$, after

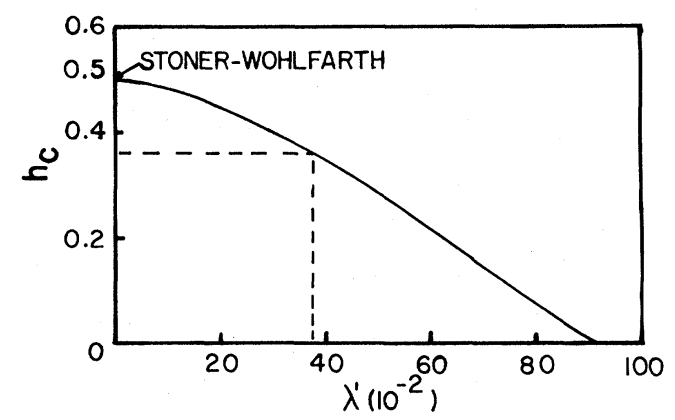

FIG. 5. Variation of coercive force with the interaction parameter $\lambda^{\prime}$. correcting for the demagnetizing field $H_{D}$ which was assumed to be $4 \pi M$ since the samples were very thin plates perpendicular to the magnetic field. This value of $\lambda^{\prime}$ was subsequently used to predict $H_{c}$ using Fig. 5 . The magnetization curve for an array of noninteracting $(\lambda=0)$ single-domain particle ${ }^{24}$ is shown in Fig. 6. Any value of remanence greater than $0.5 \mathrm{sug}$ gests the presence of interactions; in $\mathrm{Tb}_{75} \mathrm{Au}_{25}$ the measured remanence is $m_{R}=0.65$. According to Fig. 6 this implies an effective exchange field $h^{\prime}=0.25$, leading to a $\lambda^{\prime}=0.38$ since $h^{\prime}=h+\lambda^{\prime} m_{R}$. In Fig. 5, the coercive field corresponding to $\lambda^{\prime}=0.38$ is found to be $h_{c}=0.37$ and therefore

$$
H_{c}=0.75 \mathrm{~K} / M_{\mathrm{s}} \simeq 40 \mathrm{kOe},
$$

a value close to the extrapolated absolute-zero coercivity $H_{c, 0}$.

The model as discussed assumes single values of $K$ and $\lambda$. Mössbauer measurements ${ }^{25}$ tend to justify the single value of anisotropy $K$. However in reality a range of $\lambda$ values seems to be more reasonable. The microdomain model is consistent with low-anglediffraction studies ${ }^{26}$ and magnetic-aftereffect measurements $^{27}$ which show the presence of "microdomains" two orders of magnitude smaller than the conventional magnetic domains observed in ordinary ferromagnets.

In Er and Pr glasses the observed reduced remanences are smaller than 0.5 (Table I). This is not explained in this model and suggests either the existence of domain walls or the presence of antiferromagnetic interactions in these rare-earth amorphous materials.

\section{Single-ion anisotropy}

The antiferromagnetic interactions were taken into account by Ferrer et al. ${ }^{28}$ who used a molecular-field approximation and a Monte Carlo method with the

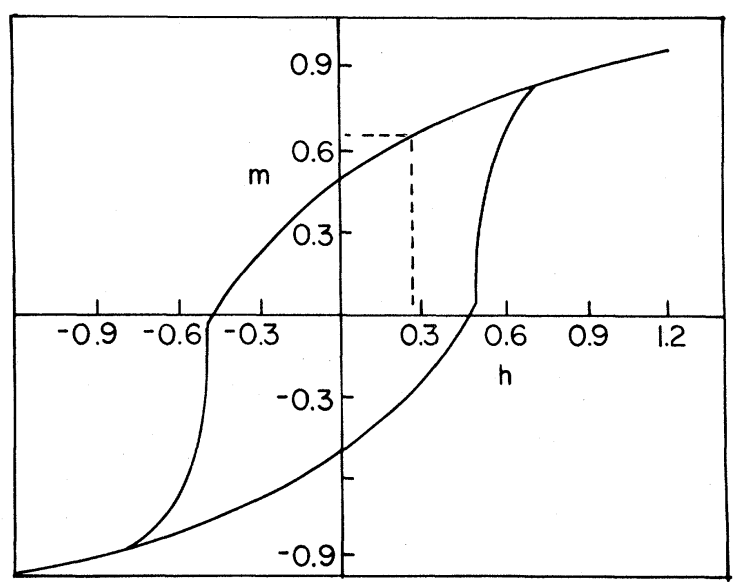

FIG. 6. Hysteresis loop for a random array of noninteracting single-domain particles. 
original Hamiltonian of the local random anisotropy model, ${ }^{9}$

$$
H=-\frac{1}{2} \mathcal{J} \sum_{\langle i, j\rangle} \overrightarrow{\mathrm{J}}(i) \cdot \overrightarrow{\mathrm{J}}(j)-D \sum_{i} J_{z_{i}}^{2}(i)-\mu H \sum_{i} J_{z}(i)
$$

where $\mathcal{J}$ is the nearest-neighbor exchange constant, $D$ the single-ion anisotropy constant $(K=n D), \mu$ the magnetic moment, and $n$ the number of spins per unit volume.

In the case of antiferromagnetic interactions the remanence was found to be always smaller than 0.5 , even approaching zero at a finite value of $D / \mathcal{J}$. Similarly, the coercive field in the theory ${ }^{27}$ was shown to be lower than that in the ferromagnetic case, disappearing at $D / \mathcal{J} \sim 2.5$. One can therefore use these figures to get values of the $D / J$ ratio from the observed remanences and use them to predict $H_{c: 0}$. In $\mathrm{Er}$ and $\mathrm{Pr}$ glasses the measured remanence is $m_{R}=0.3$ and 0.1 , respectively. This would imply a $D / \mathcal{J}$ of 5 and 3 , respectively, leading to coercive fields of 32 and $13 \mathrm{kOe}$ for the Er and Pr glasses (Table I). These coercive fields appear to be higher than the experimentally measured. However as pointed out by Boucher ${ }^{5}$ both ferromagnetic and antiferromagnetic interactions may be present in alloys of this type, leading to a more complicated magnetic structure where the application of the previous argument is too simple. In fact Harris ${ }^{16}$ has shown that the ground state in these alloys is not the "asperomagnetic" but one with about $25 \%$ of the spins reversed. The magnetization reversal could take place inhomogeneously with the reversed spins acting as nucleation centers for reversing the magnetization of the sample. This can reduce the effective exchange field and consequently the coercivity of the sample.

Finally one cannot exclude the possibility of domain walls particularly in the case of very high anisotropy. Such domain walls were used by $\mathrm{Chi}$ and Egami ${ }^{13}$ to explain the temperature dependence of coercivity in amorphous $\mathrm{TbFe}_{2}$. The thin domain walls if present could be pinned on an atomic scale by fluctuations in anisotropy and exchange interactions. The existence of such walls has not yet been proven by the micromagnetic theory. More information about the short-range atomic order and about the spatial distribution of the anisotropy axes is required in order to define the true magnetic structure.

\section{ACKNOWLEDGMENTS}

We wish to thank Simon Foner and Larry Rubin for their important contributions to the high-field measurements performed at the National Magnet Laboratory. We are grateful to Professor Paul Gaunt for informative discussions. This research was supported by the National Science Foundation under Grant No. DMR-7810781. Research above 80 kOe was performed at the National Magnet Laboratory which is supported at MIT by the National Science Foundation.
'A. E. Clark, Appl. Phys. Lett. 23, 642 (1973).

${ }^{2}$ J. J. Rhyne, J. H. Schelleng, and N. C. Koon, Phys. Rev. B 10, 4672 (1974).

3J. A. Gerber, D. J. Miller, and D. J. Sellmyer, J. Appl. Phys. 49, 1699 (1978).

${ }^{4}$ J. A. Gerber, S. G. Cornelison, W. L. Burmester, and D. J. Sellmyer, J. Appl. Phys. 50, 1608 (1979).

${ }^{5}$ B. Boucher, Phys. Status Solidi (a) 40, 197 (1977).

${ }^{6} \mathrm{~N}$. Heiman and N. Kazama, J. Appl. Phys. 49, 1686 (1978).

${ }^{7}$ D. J. Sellmyer, G. Hadjipanaysis, and S. G. Cornelison, J. Non-Cryst. Solids 40, 437 (1980).

${ }^{8}$ A. Berrada, J. Durand, N. Hassanain, and B. Loegel, J. Appl. Phys. 50, 7621 (1979).

${ }^{9}$ R. Harris, M. Plischke, and M. J. Zuckermann, Phys. Rev. Lett. 31, 160 (1973); R. W. Cochrane, R. Harris, and M.J. Zuckermann, Phys. Rep. 48, 1 (1978).

${ }^{10}$ J. M. D. Coey, J. Appl. Phys. 49, 1646 (1978).

${ }^{11}$ M. C. Chi and R. Alben, J. Appl. Phys. 48, 2987 (1977).

12J. D. Patterson, G. R. Gruzalski, and D. J. Sellmyer, Phys. Rev. B 18, 1377 (1978).

${ }^{13}$ M. C. Chi and T. Egami, J. Appl. Phys. 50, 165 (1979).

${ }^{14} \mathrm{C}$. Jayaprakash and S. Kirkpatrick, Phys. Rev. B 21, 4072 (1980)

${ }^{15}$ R. A. Pelcovits, E. Pytte, and J. Rudnick, Phys. Rev. Lett. $\underline{40,} 476$ (1978); R. A. Pelcovits, Phys. Rev. B 19,465

(1979); E. Pytte, ibid. 18, 5046 (1978).

${ }^{16} \mathrm{R}$. Harris (unpublished).

${ }^{17}$ E. Callen, Y. J. Liu, and J. R. Cullen, Phys. Rev. B $\underline{16}$, 263 (1977).

18P. Duwez, in Progress in Solid State Chemistry, edited by H. Reiss (Pergamon, Oxford, 1976), Vol. 3, p. 377.

${ }^{19} \mathrm{C}$. M. Place and P. Rhodes, J. Appl. Phys. $\underline{39}, 1282$ (1968).

${ }^{20}$ E. Kneller, Ferromagnetisms (Springer, Berlin, 1962).

${ }^{21}$ B. Boucher, IEEE Trans. Magn. 13, 1609 (1977).

${ }^{22}$ P. Gaunt (private communication).

${ }^{23}$ W. Sucksmith, Proc. R. Soc. London Ser. A 225,362 (1954).

${ }^{24}$ E.C. Stoner and E. P. Wohlfarth, Philos. Trans. R. Soc. London Ser. A $\underline{240}, 599$ (1948).

${ }^{25}$ D. Sarkar, R. Segnan, E. K. Cornell, E. Callen, R. Harris, M. Plischke, and M. J. Zuckermann, Phys. Rev. Lett. $\underline{32}$, 542 (1974).

${ }^{26}$ S. J. Pickart, J. J. Rhyne, and H. A. Alperin, Phys. Rev. Lett. 33,424 (1974)

${ }^{27}$ G. Hadjipanayis and D. J. Sellmyer, Phys. Rev. B. 23, 3355 (1981) (following paper).

${ }^{28}$ R. Ferrer, R. Harris, S. H. Sung, and M. J. Zuckerman, J. Phys. (Paris) 40, C5-221 (1979). 